\title{
Influence of Rigid Boundary and Initial Stress on the Propagation of Love Wave
}

\author{
Shishir Gupta, Amares Chattopadhyay, Sumit K. Vishwakarma, Dinesh K. Majhi \\ Department of Applied Mathematics, Indian School of Mines, Dhanbad, India \\ E-mail:shishir_ism@yahoo.com \\ Received March 10, 2011; revised March 24, 2011; accepted March 28, 2011
}

\begin{abstract}
In the present paper we study the effect of rigid boundary on the propagation of Love waves in an inhomogeneous substratum over an initially stressed half space, where the heterogeneity is both in rigidity and density. The dispersion equation of the phase velocity has been derived. It has been found that the phase velocity of Love wave is considerably influenced by the rigid boundary, inhomogeneity and the initial stress present in the half space. The velocity of Love waves have been calculated numerically as a function of $K H$ (where $K$ is a wave number $H$ is a thickness of the layer) and are presented in a number of graphs.
\end{abstract}

Keywords: Love Waves, Initial Stress, Heterogeneous Half-Space, Rigid Boundary, Phase Velocity

\section{Introduction}

The earth is a non-homogeneous medium with variations in density and rigidity in constituent layer. A study on earth structure and earth quakes $[1,2]$ says that inside the earth there exist materials which are anisotropic in nature i.e., deviate from the directionally regular elastic behaviour of an isotropic material. The study of generation and propagation of waves in layered anisotropic media, with various geometrical configurations are important in Geophysics, Seismology, Acoustic and Electromagnetism. Theoretical studies of anisotropy usually dealt with limiting case such as infinitesimal thickness and infinite wavelength, or with infinite thickness and infinite frequency. According to some seismologists anisotropy is the limiting case of a laminated solid as the laminations become infinitesimal, and Stoneley considers surface wave propagation along a half space.

The effect of inhomogeneity and rigid boundary on Love wave propagation is becoming increasingly important as seismologists study the structure of earth in ever finer details. Love waves are more sensitive to structural complexities than are Rayleigh waves. The propagation of Love waves in a medium having different types of crustal thickness was discussed by Satô [3] and De Noyer [4].

Propagation of Love waves under some particular physical conditions which are likely to exist in the interior of the earth is studied in this paper. It is Love [5], who first predicted that the earth is in a state of high initial stress. Due to atmospheric pressure, gravity vibration, creep, difference in temperature, large initial stresses may exist inside the earth. The stresses which exist in an elastic body even though external forces are absent are termed as initial stresses and the body is said to be initially stressed. These stresses might exert significant influence on the elastic waves produced by earthquakes, explorations or impacts. Thus, it is imperative to deal with the properties of wave propagation under initial stress. It was Biot [6] who first pointed out that the initial stress influences elastic waves to a great extent. The theory of incremental deformation formulated by Biot [7] in his famous book "Mechanics of Incremental Deformation" has been employed by several authors to study the propagation of surface waves in pre-stressed elastic solids. Propagation of Love waves in a non-homogeneous orthotropic elastic layer under initial stress overlying semi-infinite medium is studied by Abd-Alla and Ahmed [8]. Khurana [9] has shown the effect of initial stress on the propagation of Love wave. Wave velocities in a pre-stressed anisotropic elastic medium have been studied by Sharma and Garg [10]. References to be made to Das and Dey [11,12], Dey [13], Dey and Addy [14], Chattopadhyay and De [15], Chattopadhyay and Kar [16] and others. They suggested that the studies on the problem of elastic wave inside the earth deserve the consideration of initial stresses present in the medium. These stresses might exert significant influence on the elastic 
waves produced by earthquakes, explorations or impacts. Thus, it is imperative to deal with the problems of wave propagation under initial stress.

The near surface of the earth consists of layers of different types of material properties overlying a half space of various types of rock, underground water, oil \& gases. So, the studies of the propagation of seismic waves will be of great interest to seismologist. A detail study on elastic wave propagation and its generation in seismology had been made by Pujol [17] and Chapman [18]. In the present paper Love wave propagation in anisotropic layer of sandstone with rigid boundary over a prestressed orthotropic quartz medium has been studied. The inhomogeneity of the layer is taken into consideration by assuming $N=N_{0}(1+m z)^{2}, L=L_{0}(1+m z)^{2}$ and $\rho=\rho_{0}(1+m z)^{2}, m$ is a constant and having dimension that is inverse of length. Also the inhomogeneity of the half-space has been taken as $\mu=\mu_{1}(1+a z)$ and $\rho=\rho_{1}(1+b z)$ where $a, b$ are constants and having dimensions that are inverse of length. The initial stresses $P$ present in the inhomogeneous quartz half space also have effect in the velocity of propagation. The initial compressive stress is seen to reduce the velocity. The upper boundary plane of the layer is assumed to be rigid, and both the rigidity and the mass density of the underlying half space are assumed to increase linearly with depth.

\section{Formulation and Solution of the Problem}

Consider an inhomogeneous anisotropic layer of finite thickness $H$ over an initially stressed inhomogeneous quartz half-space. We assume that the upper surface of the crustal layer is rigid and horizontal. The $z$-axis is taken vertically downwards in the lower medium. The $x$-axis is chosen parallel to the layer in the direction of wave propagation, origin being taken at the depth $H$ below the upper surface of the layer as shown in Figure 1. $N, L$ and $\rho$ are the directional rigidities and density at any point in the layer which is assumed to be transversely isotropic with $Z$-axis as the axis of symmetry. The inhomogeneity of the layer has been taken as $N=N_{0}(1+m z)^{2}, L=L_{0}(1+m z)^{2}$ and $\rho=\rho_{0}(1+m z)^{2}$, $m$ is a constant and having dimension that is inverse of length. In the half space rigidity and density vary linearly with depth i.e. $\mu=\mu_{1}(1+a z)$ and $\rho=\rho_{1}(1+b z)$

where $a, b$ are constants and having dimensions that are inverses of length.

\subsection{Solution for the Layer}

Let $u, v$ and $w$ be the displacement components in the $x, y$ and $z$ direction respectively. Starting from the general equation of motion and using the conventional Love waves conditions viz., $u=0, w=0$ and $v=v_{1}(x, z, t)$, the only $y$ component. Then the equation of motion in absence of body force can be written as [7]

$$
N \frac{\partial^{2} v_{1}}{\partial x^{2}}+\frac{\partial}{\partial z}\left(L \frac{\partial v_{1}}{\partial z}\right)=\rho \frac{\partial^{2} v_{1}}{\partial t^{2}}
$$

For a wave propagating along $x$-direction, we may assume

$$
v_{1}=V(z) \mathrm{e}^{i K(x-c t)}
$$

Using Equation (2), Equation (1) takes the form

$$
\frac{\mathrm{d}^{2} V}{\mathrm{~d} z^{2}}+\frac{1}{L} \frac{\mathrm{d} L}{\mathrm{~d} z} \frac{\mathrm{d} V}{\mathrm{~d} z}+\frac{K^{2}}{L}\left(c^{2} \rho-N\right) V=0
$$

After putting $V=\frac{V_{1}}{L}$ in Equation (3), we get

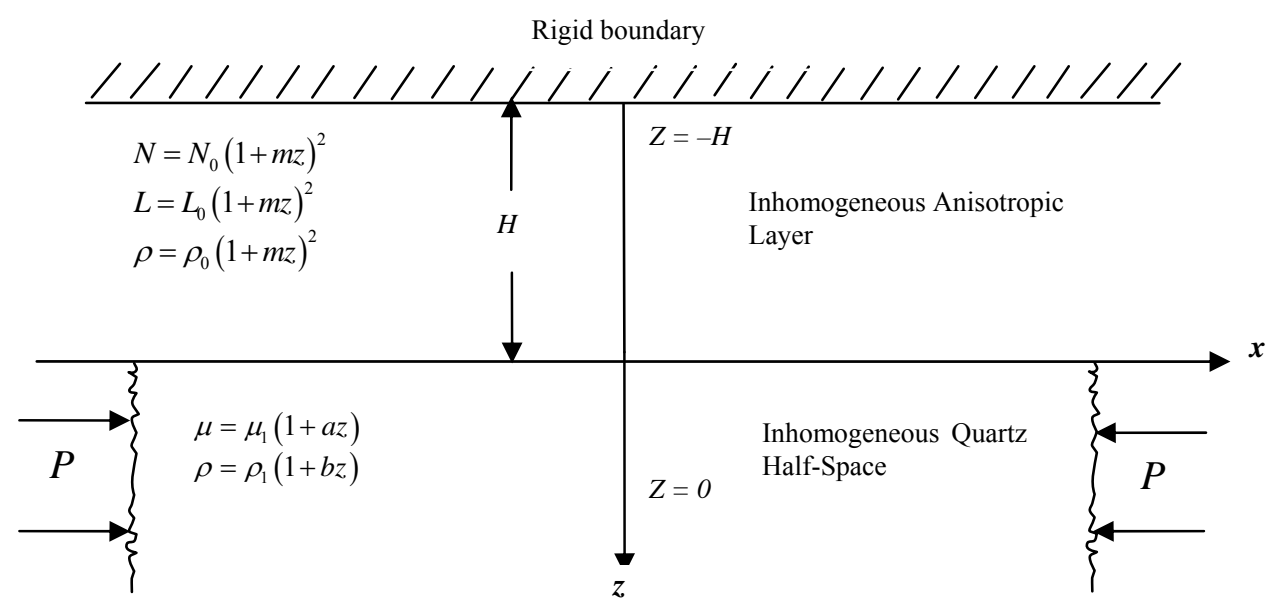

Figure 1. Geometry of the problem. 
$\frac{\mathrm{d}^{2} V_{1}}{\mathrm{~d} z^{2}}-\frac{1}{2 L} \frac{\mathrm{d}^{2} L}{\mathrm{~d} z^{2}} V_{1}+\frac{1}{4 L^{2}}\left(\frac{\mathrm{d} L}{\mathrm{~d} z}\right)^{2} V_{1}+\frac{K^{2}}{L}\left(c^{2} \rho-N\right) V_{1}=0$

The variations in rigidities and density are taken as

$N=N_{0}(1+m z)^{2}, L=L_{0}(1+m z)^{2}$ and $\rho=\rho_{0}(1+m z)^{2}$

where $m$ is a constant having dimension inverse of length.

Using Equation (5), Equation (4) changes to

$$
\frac{\mathrm{d}^{2} V_{1}}{\mathrm{~d} z^{2}}+m_{1}^{2} V_{1}=0
$$

where

$$
m_{1}^{2}=\frac{K^{2}}{L_{0}}\left(c^{2} \rho_{0}-N_{0}\right)
$$

The solution of Equation (6) may be assumed as

$$
V_{1}=A \mathrm{e}^{i m_{1} z}+B \mathrm{e}^{-i m_{1} z}
$$

Thus the solution for the non-homogeneous, anisotropic layer may be taken as

$$
v_{1}=\frac{A \mathrm{e}^{i m_{1} z}+B \mathrm{e}^{-i m_{1} z}}{\sqrt{L_{0}}(1+m z)} \mathrm{e}^{i K(x-c t)}
$$

\subsection{Solution for the Half-Space}

The lower medium is considered as inhomogeneous quartz half-space under initial horizontal compressive stress $P$ along $x$-axis. The eqn. of motion corresponding to the displacement due to Love waves can be written as [7]

$$
\frac{\partial s_{21}}{\partial x}+\frac{\partial s_{23}}{\partial z}-\frac{P}{2}\left(\frac{\partial^{2} v}{\partial x^{2}}\right)=\frac{\partial^{2}}{\partial t^{2}}\left(\rho v_{2}\right)
$$

where $s_{i j}$ are the incremental stress components in the half-space, $P$ is the initial compressive stress along the $x$-direction, and $\rho$ is the density of the material of the half space. In the present problem we have

$$
\mu=\mu_{1}(1+a z) \text { and } \rho=\rho_{1}(1+b z)
$$

Using the stress-strain relations

$$
s_{21}=2 \mu e_{x y}, s_{23}=2 \mu e_{y z}
$$

And the relation (10), the equation of motion (9) can be written as

$$
\left[1-\frac{P}{2 \mu_{1}(1+a z)}\right] \frac{\partial^{2} v_{2}}{\partial x^{2}}+\frac{\partial^{2} v_{2}}{\partial z^{2}}+\frac{a}{1+a z} \frac{\partial v_{2}}{\partial z}=\frac{\rho_{1}(1+b z)}{\mu_{1}(1+a z)} \frac{\partial^{2} v_{2}}{\partial t^{2}}
$$

Let

$$
\begin{array}{cr}
v_{2}=V(z) \mathrm{e}^{i K(x-c t)} & \text { (13) } \\
\frac{\mathrm{d}^{2} V}{\mathrm{~d} z^{2}}+\frac{a}{1+a z} \frac{\mathrm{d} V}{\mathrm{~d} z}+\left[\frac{\rho_{1}(1+b z)}{\mu_{1}(1+a z)} c^{2}-\left\{1-\frac{P}{2 \mu_{1}(1+a z)}\right\}\right] K^{2} V=0
\end{array}
$$

Using Equation (13), Equation (12) takes the form

Putting $V=\frac{\phi(z)}{(1+a z)^{1 / 2}}$ in Equation (14) to eliminate the term $\frac{d V}{d z}$, we get

$$
\phi^{\prime \prime}(z)+\left[\frac{a^{2}}{4(1+a z)^{2}}-K^{2}\left\{\left(1-\frac{P}{2 \mu_{1}(1+a z)}\right)-\frac{c^{2}}{c_{o s}^{2}} \frac{(1+b z)}{(1+a z)}\right\}\right] \phi(z)=0
$$

where $c_{o s}=\sqrt{\frac{\mu_{1}}{\rho_{0}}}$ and $c$ is phase velocity.

Substituting

$$
\gamma_{1}=\left[1-\frac{P}{2 \mu_{1}(1+a z)}-\frac{c^{2}}{c_{o s}^{2}} \frac{b}{a}\right]^{1 / 2}, \eta=\frac{2 \gamma_{1} K(1+a z)}{a}, \omega=K c
$$

in Equation (15) we get

$$
\frac{\mathrm{d}^{2} \phi}{\mathrm{d} \eta^{2}}+\left[\frac{R}{2 \eta}+\frac{1}{4 \eta^{2}}-\frac{1}{4}\right] \phi(\eta)=0
$$

where $R=\frac{\omega^{2}(a-b)}{c_{o s}^{2} \gamma_{1} a^{2} K}$ Equation (16) is a Whittaker's equation, solution of which may be written as 


$$
\phi(\eta)=D_{1} W_{R / 2,0}(\eta)+D_{2} W_{-R / 2,0}(-\eta)
$$

where $D_{1}$ and $D_{2}$ are arbitrary constants and $W_{R / 2,0}(\eta)$ is the Whittaker's function. The solution of Equation (16) satisfying the condition $\lim z \rightarrow \infty$ when $V(z) \rightarrow 0$ i.e. $\lim \eta \rightarrow \infty$ when $\phi(\eta) \rightarrow 0$ may be taken as

$$
\phi(\eta)=D_{1} W_{R / 2,0}(\eta)
$$

$$
v_{2}=D_{1} \mathrm{e}^{\frac{-\gamma_{1} K(1+a z)}{a}}\left\{\frac{2 \gamma_{1}(1+a z)}{a}\right\}^{R / 2}\left[\frac{1}{(1+a z)^{1 / 2}}-\frac{\left(\frac{R-1}{2}\right)^{2} a}{2 \gamma_{1} K(1+a z)^{3 / 2}}\right] \mathrm{e}^{i K(x-c t)}
$$

\section{Boundary Conditions and Dispersion equation}

At the interface, $z=0$ the continuity of the stress requires that $\left(\tau_{y z}\right)_{1}=\left(\Delta f_{y}\right)_{2}$ At rigid boundary $z=-H$, the displacement is vanishing so that $v_{1}=0$, at $z=-H$ and the continuity of the displacement requires that $v_{1}=v_{2}$ at $Z=0$

where $\Delta f_{y}$ is the incremental boundary force per unit initial area in the pre-stressed half-space at deformed stage, the physical explanations of which may be obtained from Figure 2. In the boundary conditions the quantities (i.e. $\Delta f_{y}$ and $v$ ) for the stratum (inhomogeneous and anisotropic) and lower half-space are denoted by the subscripts 1 and 2 respectively.

The magnitude of $\Delta f_{y}$ as derived by [7] is

$$
\Delta f_{i}=\left(s_{i j}+S_{k j} w_{i k}+S_{i j} e-S_{i k} e_{j k}\right) n_{j}
$$

where $S_{i j}$ are the initial stress components, $S_{i j}$ are the incremental stress components, $e_{i j}$ are the strain components, $w_{i j}$ are the rotational components, $e$ is the dilatation and $n_{j}$ is the cosine of angle between the $j^{\text {th }}$ direction and normal to the surface. It is obvious that
$\Delta f_{z}$ is the incremental normal force per unit area to the boundary whereas, $\Delta f_{x}$ and $\Delta f_{y}$ are shear forces. In the present problem, since $S_{11}$ is the only initial stress component, $v_{2}=v_{2}(x, z, t), u_{2}=0, w_{2}=0$ and also $z$ - axis is normal to the boundary.

$$
\Delta f_{y}=s_{23}=\mu \frac{\partial v_{2}}{\partial z}
$$

Using boundary conditions (20) in Equation (8) and Equation (19), we get

$$
\begin{gathered}
A\left(\sqrt{L_{0}} i m_{1}-m \sqrt{L_{0}}\right)-B\left(\sqrt{L_{0}} i m_{1}+m \sqrt{L_{0}}\right)-D_{1} K_{1}=0 \\
A \mathrm{e}^{-i m_{1} H}+B \mathrm{e}^{i m_{1} H}=0 \\
A+B-D_{1} \sqrt{L_{0}} K_{2}=0
\end{gathered}
$$

where

$$
\begin{aligned}
& K_{1}=\mathrm{e}^{\frac{-\gamma_{1} K}{a}}\left(\frac{2 \gamma_{1}}{a}\right)^{R / 2} \mu_{0}\left[\frac{-a}{2}+\frac{3 a}{2} \frac{\left(\frac{R-1}{2}\right)^{2} a}{2 \gamma_{1} K}+\left\{1-\frac{\left(\frac{R-1}{2}\right)^{2} a}{2 \gamma_{1} K}\right\} \cdot\left\{\frac{a R}{2}-\gamma_{1} K\right\},\right. \\
& K_{2}=\mathrm{e}^{\frac{-\gamma_{1} K}{a}}\left(\frac{2 \gamma_{1}}{a}\right)^{R / 2}\left\{1-\frac{\left(\frac{R-1}{2}\right)^{2} a}{2 \gamma_{1} K}\right\}
\end{aligned}
$$

Eliminating $A, B$ and $D_{1}$ from Equations (21)-(23), we get

$$
\mid \begin{aligned}
& \sqrt{L_{0}} i m_{1}-m \sqrt{L_{0}} \\
& e^{-i m_{1} H} \\
& 1
\end{aligned}
$$

$$
\begin{gathered}
-K_{1} \\
0 \\
-\sqrt{L_{0}} K_{2}
\end{gathered} \mid=0
$$




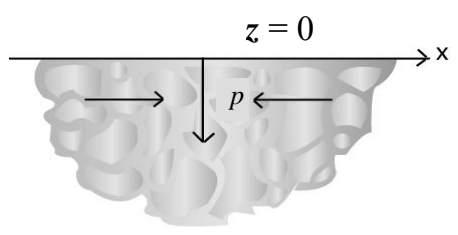

(a)

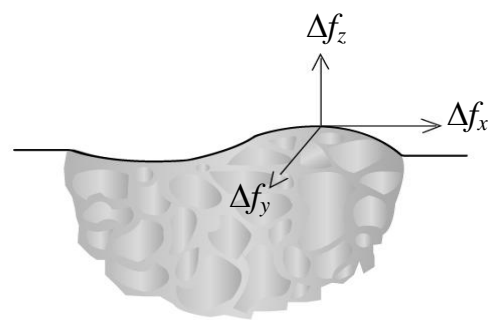

(b)

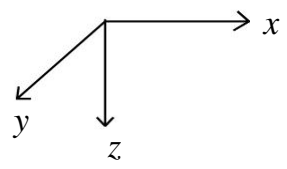

(c)

Figure 2. Incremental boundary forces.

Expanding the above determinant and solving, we get

$$
\cot \left[K H \sqrt{\frac{c^{2} \rho_{0}-N_{0}}{L_{0}}}\right]=\frac{K_{1}+L_{0} K_{2} m}{L_{0} K_{2} m_{1}}
$$

Putting the values of $K_{1}, K_{2}$ and $m_{1}$ in Equation (24),

we get

$$
\cot \left[K H \sqrt{\frac{c^{2}}{c_{0}^{2}}-\frac{N_{0}}{L_{0}}}\right]=\frac{A_{1}}{A_{2}}
$$

where

$$
\begin{aligned}
& A_{1}=L_{0} m\left\{1-\frac{\left(\frac{R-1}{2}\right)^{2} a}{2 \gamma_{1} K}\right\}+\mu_{1}\left[\frac{-a}{2}+\frac{3 a^{2}}{2} \frac{\left(\frac{R-1}{2}\right)^{2}}{2 \gamma_{1} K}+\left\{1-\frac{\left(\frac{R-1}{2}\right)^{2} a}{2 \gamma_{1} K}\right\}\left\{\frac{a R}{2}-\gamma_{1} K\right\}\right] \\
& A_{2}=L_{0} K \sqrt{\frac{\left(c^{2} \rho_{0}-N_{0}\right)}{L_{0}}}\left\{1-\frac{\left(\frac{R-1}{2}\right)^{2} a}{2 \gamma_{1} K}\right\}
\end{aligned}
$$

$c_{0}=\sqrt{\frac{L_{0}}{\rho_{0}}}, \gamma_{1}=\left[1-\zeta-\frac{c^{2}}{c_{o s}^{2}} \cdot \frac{b}{a}\right]^{1 / 2}$ and $\zeta=\frac{P}{2 \mu_{1}}$ is the non-dimensional parameter due to initial stress $P$.

The Equation (25) gives the phase velocity of Love wave in an inhomogeneous anisotropic layer over an initially stressed inhomogeneous half space when the upper boundary plane of the layer is assumed to be rigid.

\section{Particular Cases}

Case 1

$$
\begin{aligned}
& \left.A_{1}=L_{0} m\left\{1-\frac{\left(\frac{R-1}{2}\right)^{2} a}{2 \gamma_{1} K}\right\}+\mu_{1} \frac{-a}{2}+\frac{3 a^{2}}{2} \frac{\left(\frac{R-1}{2}\right)^{2}}{2 \gamma_{1} K}+\left\{1-\frac{\left(\frac{R-1}{2}\right)^{2} a}{2 \gamma_{1} K}\right\}\left\{\frac{a R}{2}-\gamma_{1} K\right\}\right] \\
& A_{2}=L_{0} K \sqrt{\frac{\left(c^{2} \rho_{0}-N_{0}\right)}{L_{0}}}\left\{1-\frac{\left(\frac{R-1}{2}\right)^{2} a}{2 \gamma_{1} K}\right\}
\end{aligned}
$$

If the half-space is taken as Gibson half-space i.e., rigidity varies linearly with depth whereas the density remains constant. In this case $a \neq 0$ but $b=0$ so that $\rho=\rho_{1}$ (constant) and the dispersion Equation (25) takes the form

$$
\cot \left[K H \sqrt{\frac{c^{2}}{c_{0}^{2}}-\frac{N_{0}}{L_{0}}}\right]=\frac{A_{1}}{A_{2}}
$$

where 


$$
\gamma_{1}=[1-\zeta]^{1 / 2}, \quad R=\frac{\omega^{2}}{c_{o s}^{2} \gamma_{1} a K}
$$

which is the dispersion equation of Love wave in an inhomogeneous layer lying over an initially stressed Gibson half space, when the upper boundary plane of the layer is assumed to be rigid

\section{Case 2}

If $a \neq 0, p \neq 0, b=0, m=0$ then the dispersion Equation (25) takes the form

$$
\cot \left[K H \sqrt{\frac{c^{2}}{c_{0}^{2}}-\frac{N_{0}}{L_{0}}}\right]=\frac{A_{1}}{A_{2}}
$$

where

$$
\begin{aligned}
& A_{1}=L_{0} m\left\{1-\frac{\left(\frac{R-1}{2}\right)^{2} a}{2 \gamma_{1} K}\right\}+ \\
& \mu_{1}\left[\frac{-a}{2}+\frac{3 a^{2}}{2} \frac{\left(\frac{R-1}{2}\right)^{2}}{2 \gamma_{1} K}+\left\{1-\frac{\left(\frac{R-1}{2}\right)^{2} a}{2 \gamma_{1} K}\right\}\left\{\frac{a R}{2}-\gamma_{1} K\right\}\right] \\
& A_{2}=L_{0} K \sqrt{\frac{\left(c^{2} \rho_{0}-N_{0}\right)}{L_{0}}}\left\{1-\frac{\left(\frac{R-1}{2}\right)^{2} a}{2 \gamma_{1} K}\right\}
\end{aligned}
$$

which is the dispersion equation of Love wave in a homogeneous layer over an initially stressed half space with constant density and when upper boundary plane is assumed to be rigid.

\section{Case 3}

If $a \rightarrow 0, b \rightarrow 0, P \rightarrow 0$ and $m \rightarrow 0$ then the dispersion Equation (25) takes the form

$$
\cot \left[K H \sqrt{\frac{c^{2}}{c_{0}^{2}}-\frac{N_{0}}{L_{0}}}\right]=\frac{A_{1}}{A_{2}}
$$

where $A_{1}=-\mu_{1} K$ and $A_{2}=L_{0} K \sqrt{\frac{\left(c^{2} \rho_{0}-N_{0}\right)}{L_{0}}}$

which is the dispersion equation of Love wave in a homogeneous layer over a homogeneous half-space, when the upper boundary plane is assumed to be rigid and halfspace is free from initial stress.

\section{Case 4}

If $a \rightarrow 0, b \rightarrow 0, P \rightarrow 0$ and $m \rightarrow 0$ and

$N_{0}=L_{0}$ then the dispersion Equation (25) takes the form

$$
\tan \left[K H \sqrt{\frac{c^{2}}{c_{o}^{2}}-1}\right]=\frac{L_{0} \sqrt{1-\frac{c^{2}}{c_{o}^{2}}}}{\mu_{1} \sqrt{\frac{c^{2}}{c_{o s}^{2}}-1}}
$$

Equation (27) gives the phase velocity of Love wave in a homogeneous isotropic layer over a homogeneous half space when the upper boundary plane of the layer is assumed to be rigid and half space has no initial stress.

Note that the equation for the phase velocity $c$ of the Love waves in a layer overlaying a half-space, when the upper boundary plane is not rigid, is given by

$$
\tan \left[K H \sqrt{\frac{c^{2}}{c_{0}^{2}}-1}\right]=\frac{\mu_{1} \sqrt{1-\frac{c^{2}}{c_{1}^{2}}}}{L_{0} \sqrt{\frac{c^{2}}{c_{o}^{2}}-1}}
$$

It can be seen from Equation (27) and Equation (28) that the phase velocity of Love waves in a layer with a rigid surface is different from that in a layer with a free surface.

\section{Numerical Computation and Discussion}

In order to show the effect of rigid boundary, anisotropy, non-homogeneity and initial stresses on the propagation of Love waves, numerical computation of Equation (25) were performed with different values of parameter (Table 1) representing the above characteristics. The value of $c / c_{o s}$ and $m / K$ are taken as 0.7 and 0.2 respectively in all the figure except Figures 9 and 10. In Figures 3-5 curves are plotted when both density as well as rigidity varies linearly with depth in presence of rigid boundary plane. Unlike to this, Figures 6-8 have been plotted when rigidity varies linearly with depth but density remains constant, thus giving dispersion curve in Gibson half space.

Figures 3 and $\mathbf{6}$ gives the dispersion curves in the absence of initial stress, shows that the velocity of Love wave decreases rapidly when the values of $K H$ increases under the effect of rigid boundary and initial stress. This also reflects that the velocity of Love wave is finite in the vicinity of the surface of the half-space and vanishes as depth increases for a particular wave number. Moreover, in the presence of rigid boundary, it has been found that the velocity of Love wave increases for a particular value of $\mathrm{KH}$ when compared with the case of a layer having free surface.

Figures 4 and 5 show the effect of initial stress present in the half-space. It has been observed that an increase in compressive initial stresses $(\zeta>0)$ deceases the velocity of Love waves for the same frequency. The tensile initial stress $(\zeta<0)$ of small magnitude in the half-space increase the velocity, but the large magnitude of tensile stress $\zeta$ doesn't allow Love wave to propagate. Figures 6 and 7 show the influence of tensile initial stress in Gibson half-space.

Figure 9 gives the velocity of Love waves for 
Table 1. Values of various dimensionless parameters.

\begin{tabular}{|c|c|c|c|c|c|c|}
\hline Figure no. & Curve no. & $a / K$ & $b / K$ & $\zeta$ & $\mu_{1} / L_{0}$ & $N_{0} / L_{0}$ \\
\hline \multirow{4}{*}{3} & 1 & 0.1 & 0.1 & 0.0 & 0.2 & 0.1 \\
\hline & 2 & 0.1 & 0.1 & 0.0 & 0.4 & 0.2 \\
\hline & 3 & 0.1 & 0.1 & 0.0 & 0.6 & 0.3 \\
\hline & 4 & 0.1 & 0.1 & 0.0 & 0.8 & 0.4 \\
\hline \multirow{4}{*}{4} & 1 & 0.2 & 0.01 & 0.0 & 2.5 & 1.5 \\
\hline & 2 & 0.2 & 0.01 & 0.1 & 2.5 & 1.5 \\
\hline & 3 & 0.2 & 0.01 & 0.2 & 2.5 & 1.5 \\
\hline & 4 & 0.2 & 0.01 & 0.3 & 2.5 & 1.5 \\
\hline \multirow{4}{*}{5} & 1 & 0.2 & 0.01 & 0.0 & 2.5 & 1.5 \\
\hline & 2 & 0.2 & 0.01 & -0.1 & 2.5 & 1.5 \\
\hline & 3 & 0.2 & 0.01 & -0.2 & 2.5 & 1.5 \\
\hline & 4 & 0.2 & 0.01 & -0.3 & 2.5 & 1.5 \\
\hline \multirow{4}{*}{6} & 1 & 0.1 & 0.0 & 0.0 & 0.2 & 0.1 \\
\hline & 2 & 0.1 & 0.0 & 0.0 & 0.4 & 0.2 \\
\hline & 3 & 0.1 & 0.0 & 0.0 & 0.6 & 0.3 \\
\hline & 4 & 0.1 & 0.0 & 0.0 & 0.8 & 0.4 \\
\hline \multirow{4}{*}{7} & 1 & 0.2 & 0.0 & 0.0 & 2.5 & 1.5 \\
\hline & 2 & 0.2 & 0.0 & 0.1 & 2.5 & 1.5 \\
\hline & 3 & 0.2 & 0.0 & 0.2 & 2.5 & 1.5 \\
\hline & 4 & 0.2 & 0.0 & 0.3 & 2.5 & 1.5 \\
\hline \multirow{4}{*}{8} & 1 & 0.1 & 0.0 & 0.0 & 2.5 & 1.5 \\
\hline & 2 & 0.1 & 0.0 & -0.1 & 2.5 & 1.5 \\
\hline & 3 & 0.1 & 0.0 & -0.2 & 2.5 & 1.5 \\
\hline & 4 & 0.1 & 0.0 & -0.3 & 2.5 & 1.5 \\
\hline
\end{tabular}

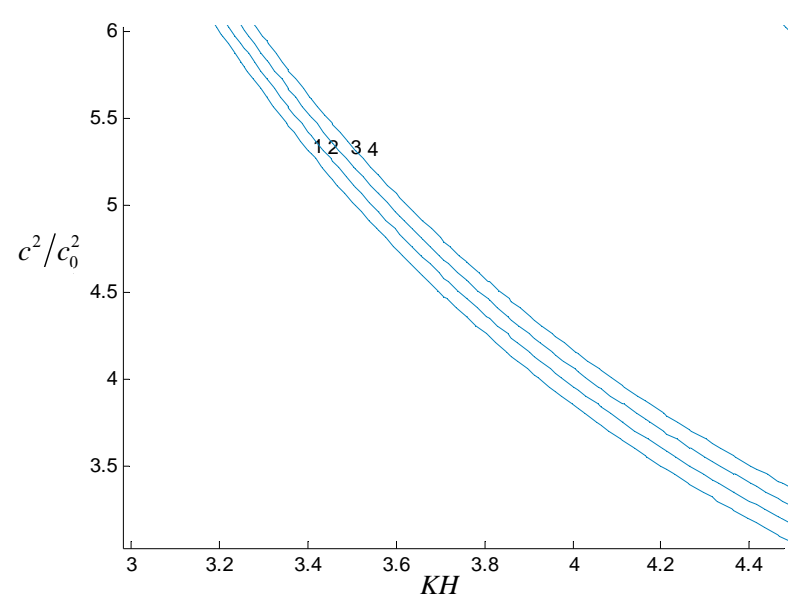

Figure 3. Dipersion curve in the absence of initial stress under the effect of rigid boundary.

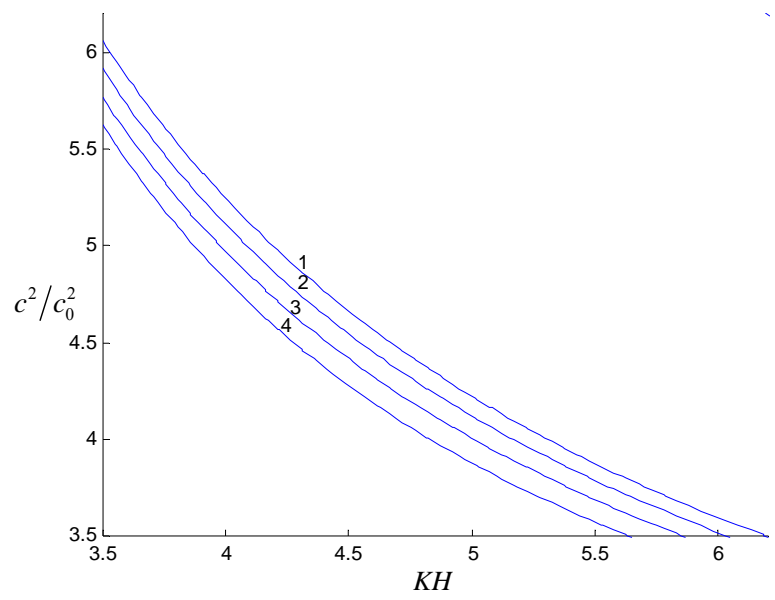

Figure 4. Dispersion curve under the effect of initial stress $(\zeta>0)$ and rigid boundary .

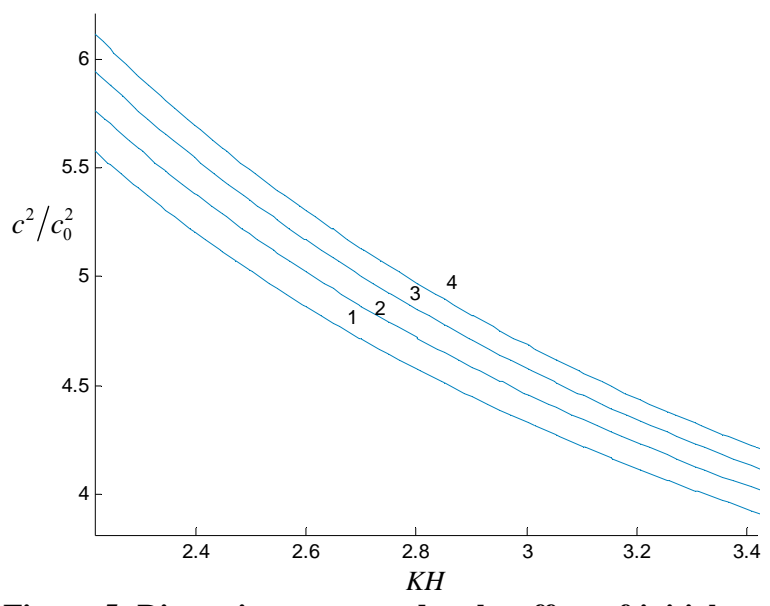

Figure 5. Dispersion curve under the effect of initial stress $(\zeta<0)$ and rigid boundary.

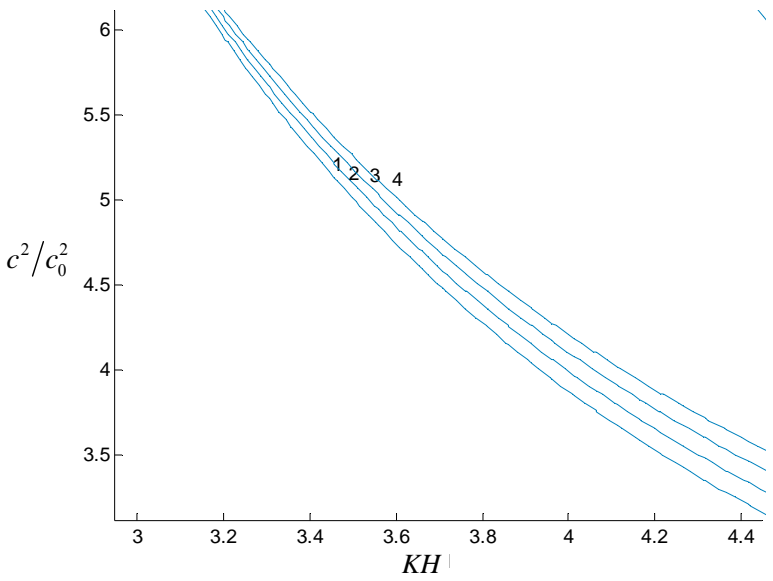

Figure 6. Dipersion curve in the absence of initial stress under the effect of rigid boundary. 


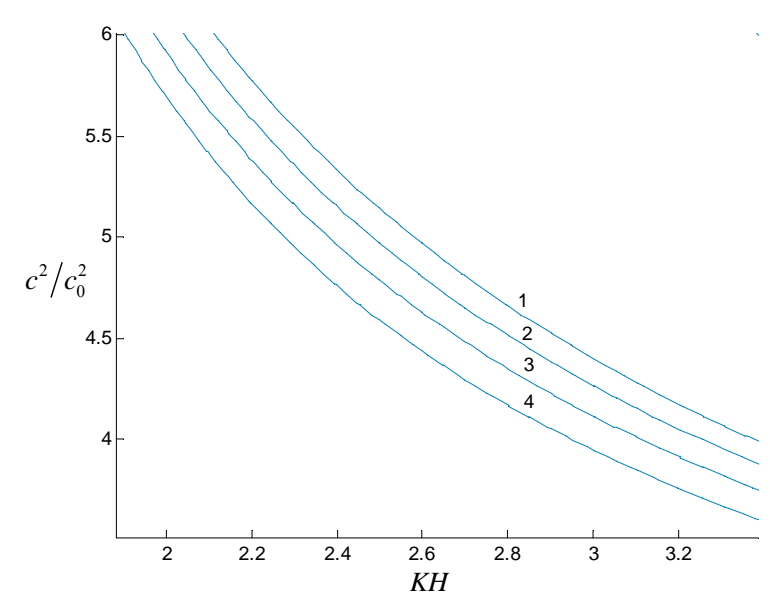

Figure 7. Dispersion curve under the effect of initial stress $(\zeta>0)$ and rigid boundary .

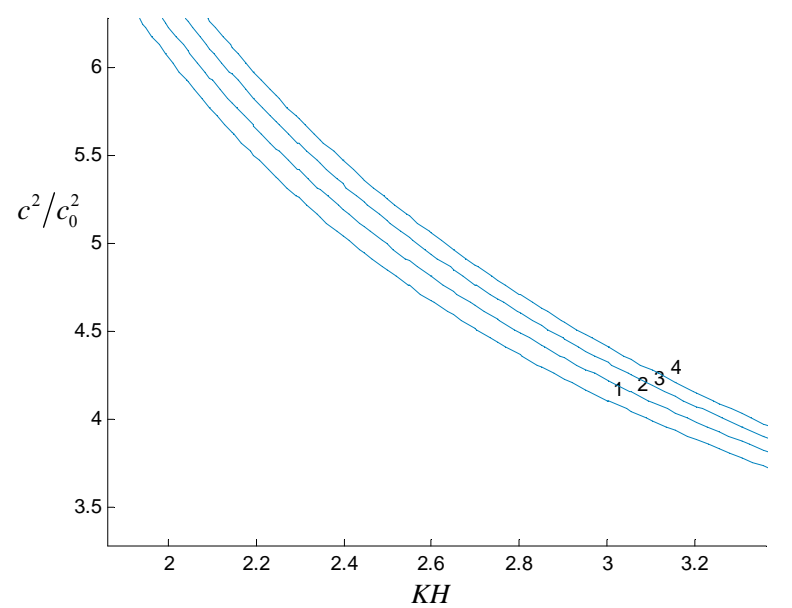

Figure 8. Dispersion curve under the effect of initial stress $(\zeta<0)$ and rigid boundary.

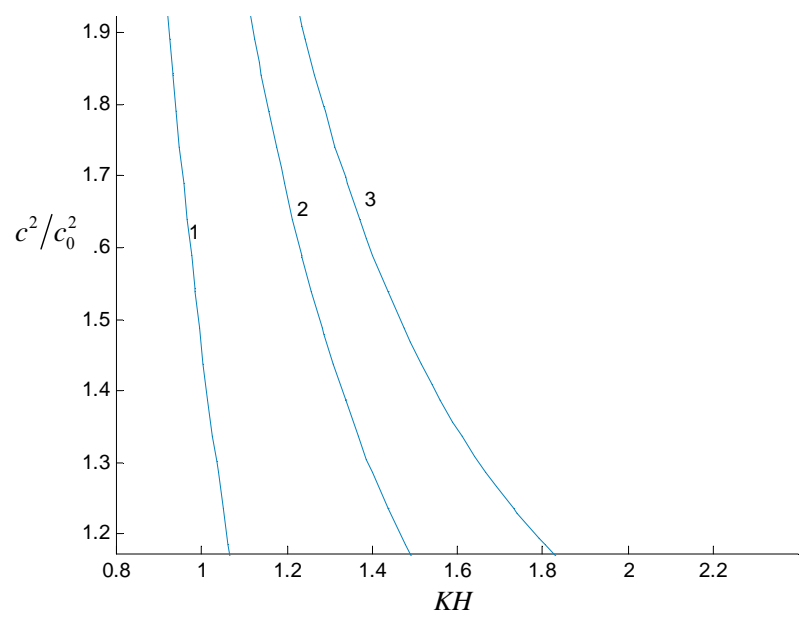

Figure 9. Dimensionless phase speed $c^{2} / c_{0}^{2}$ as function of dimensionless $K H$ evaluated from Equation (27) for $L_{0} / \mu_{1}=1.0,1.5,2.0$ and $\left(c_{0} / c_{\text {os }}\right)^{2}=0.2$.

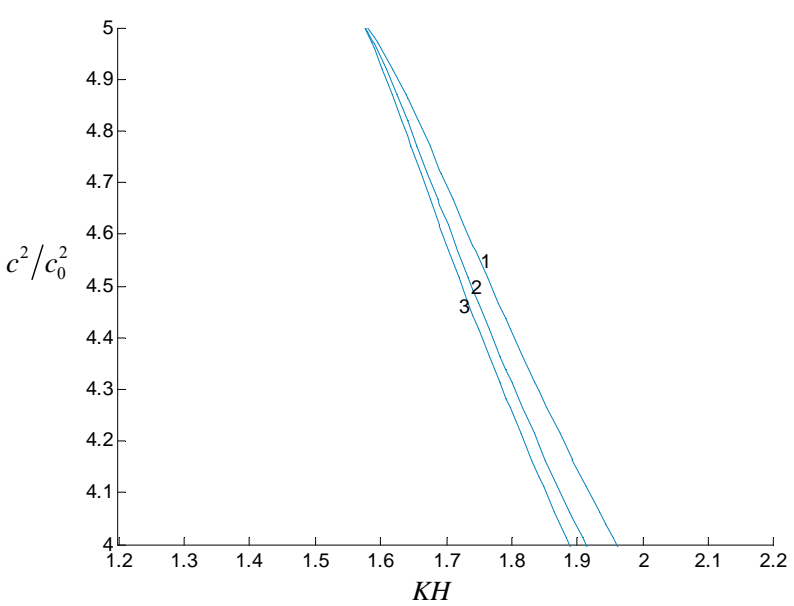

Figure 10. Dimensionless phase speed $c^{2} / c_{0}^{2}$ as function of dimensionless $K \boldsymbol{H}$ evaluated from Equation (28) for

$L_{0} / \mu_{1}=1.0,1.5,2.0$ and $\left(c_{0} / c_{\text {os }}\right)^{2}=0.2$.

$\left(c_{0} / c_{1}\right)^{2}=0.2$ in a layer over a homogeneous half-space when the upper boundary plane of the layer is assumed to be rigid. While Figure 10 gives the speed of Love waves for $\left(c_{0} / c_{1}\right)^{2}=0.2$ in a homogeneous layer over a homogeneous half-space in the absence of rigid boundary plane. It is observed that overburden layer has a prominent effect. It is also observed that magnitudes of speed are changed in entire range of $K H$ along with reversing the positions of the curve.

\section{Acknowledgements}

The author conveys their sincere thanks to Indian School of Mines, Dhanbad, for providing JRF, Mr. Sumit Kumar Vishwakarma and also facilitating us with best facility. We are also grateful to the honourable referees for their invaluable suggestions for improving the manuscript.

\section{References}

[1] S. Stein and M. Wysession, "An Introduction to Seismology, Earthquakes and Earth Structure," Blackwell Publishing, Oxford, 2003.

[2] P. M. Shearer, "Introduction to Seismology," 2nd Edition, Cambridge University Press, Cambridge, 2009.

[3] Y. Satô, "Study on Surface Waves VI: Generation of Love and Other Type of SH-Waves," Departmental Bulletin Paper, Bulletin Earthquake Research Institute, University of Tokyo, Tokyo, 1952, pp.101-120.

[4] J. D. Noyer, "The Effect of Variations in Layer Thickness on Love Waves," Bulletin of Seismology Society of America, Vol. 51, No. 2, 1961, pp. 227-235.

[5] A. E. H. Love, "A Treatise on Mathematical Theory of Elasticity," 4th Edition, Dover Publication, New York, 
1944.

[6] M. A. Biot, "The Influence of Initial Stress on Elastic Wave," Journal of Appied Physics, Vol. 11, No. 8, 1940, pp. 522-530. doi:10.1063/1.1712807

[7] M. A. Biot, "Mechanics of Incremental Deformation," John Wiley and Sons, New York, 1965.

[8] A. M. Abd-Alla and S. M. Ahmed, "Propagation of Love Waves in a Non-Homogeneous Orthotropic Elastic Layer under Intial Stress Overlying Semi-Infinite Medium," Applied Mathematics and Computation, Vol. 106, No. 2, 1999, pp. 265-275. doi:10.1016/S0096-3003(98)10128-5

[9] P. Khurana, "Love Wave Propagation in a Prestressed Medium," Indian Journal of Pure and Applied Mathematics, Vol. 32, No. 8, 2001, pp. 1201-1207.

[10] M. D. Sharma and N. Garg, "Wave Velocities in a Prestressed Anisotropic Elastic Medium," Journal of Earth System Science, Vol. 115, No. 2, 2007, pp. 257-265.

[11] S. C. Das and S. Dey, "Note on Gravity Waves," Indian Journal of Engineering Mathematics, Vol. 1, No. 2, 1968, pp. 155-160.

[12] S. C. Das and S. Dey, "Edge Waves under Initial Stress," Applied Scientific Research, Vol. 22, No. 1, 1970, pp. 382-389.
[13] S. Dey, "Wave Propagation in Two-Layered Medium under Initial Stresses," Journal of Pure and Applied Geophysics Switzerland, Vol. 90, No. 1, 1971, pp. 38-52. doi:10.1007/BF00875507

[14] S. Dey and S. K. Addy, "Love Waves under Initial Stresses in a Visco-Elastic Medium Overlying an Elastic Half-Space," Gerlands Beitr ge Zur Geophysik, Vol. 87, No. 4, 1978, pp. 306-311.

[15] A. Chattopadhyay and R. K. De, "Propagation of Love Type Waves in a Visco Elastic Initially Stressed Layer Overlying a Visco-Elastic Half-Space with Irregular Interface," Revue Roumaine Des Sciences Techniques-Series De Mechanique Appliquee, Vol. 26, No. 3, 1981, pp. 449-460.

[16] A. Chttopadhyay and B. K. Kar, "On the Dispersion Curves of Love Type Waves in an Initially Stressedcrustal Layer Having an Irregular Interface," Geophysical Research Bulletin, Vol. 16 No. 1, 1978, pp. 13-23.

[17] J. Pujole, "Elastic Wave Propagation and Generation in Seismology," Cambridge University Press, Cambridge, 2003. doi:10.1017/CBO9780511610127

[18] C. Chapman, "Fundamentals of Seismic Wave Propagation," Cambridge University Press, Cambridge, 2004. doi:10.1017/CBO9780511616877

\section{Appendix}

$\begin{array}{ll}H & \text { Thickness of the layer } \\ N, L & \text { Directional rigidities of the layer } \\ \mu & \text { Rigidity of the half-space } \\ \rho & \text { Density of the medium } \\ P & \text { Initial stress } \\ u, v, w & \text { Displacement components in radial, circumferential \& axial directions respectively } \\ K & \text { wave number } \\ C & \text { Velocity of love wave in the layer } \\ c_{0} & \text { Velocity of shear wave in the layer } \\ c_{o s} & \text { Velocity of shear wave in the half-space } \\ \eta, \gamma, R & \text { Dimensionless quantity } \\ m, a, b & \text { Constants having dimensions that are inverse of length } \\ D_{1}, D_{2}, A, B, m_{1} & \text { Arbitrary constants }\end{array}$

